\title{
HEPATITIS
}

\section{Alcohol has no effect on hepatitis $C$ virus replication: a meta-analysis}

\section{B S Anand, J Thornby}

See end of article for authors' affiliations .....................

Correspondence to: Professor B S Anand, Digestive Diseases Section (1 $11 \mathrm{D})$, VA Medical Center, 2002 Holcombe Blvd., Houston, TX 77030, USA; ana0@flash.net

Revised version received 11 March 2005

Accepted for publication 18 March 2005
Background: Patients with chronic hepatitis $\mathrm{C}$ virus (HCV) infection who consume large quantities of alcohol have more severe liver disease compared with HCV patients without a history of alcohol consumption. The mechanism by which alcohol worsens HCV related liver disease is not properly understood. One possibility is that alcohol stimulates $\mathrm{HCV}$ replication, and the present meta-analysis was performed to examine this issue.

Methods: The effect of alcohol on viral titres was assessed in three ways: comparison of the heaviest drinkers with non-drinkers; effect of graded doses of alcohol; and effect of abstinence in the same individual.

Results: A total of 14 studies were identified. Comparison of patients with the highest alcohol use with the abstinent group showed a significant association with viral load in three studies, five studies had a positive direction, while the remaining four studies found a negative relationship. Analysis of the combined results showed no association between alcohol consumption and virus levels $(p=0.29)$. Assessment of graded doses of alcohol also showed no significant difference between non-drinkers and moderate drinkers $(p=0.50)$, between non-drinkers and heavy drinkers $(p=0.35)$, or between moderate drinkers and heavy drinkers $(p=0.32)$. Five studies examined the influence of abstinence on viral titres but none provided sufficient data for statistical analysis.

Conclusions: The present study has failed to show an association between alcohol use and HCV viral titres. These observations raise the possibility that the hepatic damage caused by alcohol and HCV may be purely additive, involving different mechanisms and pathways.
$\mathrm{N}$ early $75-85 \%$ of people exposed to the hepatitis $C$ virus (HCV) develop chronic infection. ${ }^{12}$ Disease progression in HCV related liver disease is extremely variable. In most subjects the disease runs an indolent course and it may take several decades for the development of significant liver disease. Indeed, many individuals never experience liver disease and die of unrelated causes. At the other end of the spectrum, patients have an aggressive illness, and may develop end stage disease within a few years. For example, HCV related liver damage runs a more aggressive course in immunocompromised patients such as those with human immunodeficiency virus infection and after liver transplantation. $^{3}$ In general, it is not possible to predict the course of illness in a particular individual although several factors have been identified that may impact on disease progression. Male sex and older age at the time of infection are associated with a faster rate of progression. ${ }^{4-7}$ African Americans have a lower rate of hepatic fibrosis compared with Caucasians, ${ }^{89}$ despite a higher rate of chronic infection ${ }^{1}$ and lower response to antiviral therapy. ${ }^{10}$ Coinfection with hepatitis $\mathrm{B}$ virus is associated with more rapid onset of cirrhosis, decompensated liver disease, and hepatocellular carcinoma. ${ }^{11}{ }^{12}$

Another factor that is strongly implicated in the severity of HCV related liver disease is alcohol consumption. There is a higher rate of HCV infection in chronic alcohols compared with the general population. ${ }^{13-16}$ HCV infected chronic alcoholics have more severe clinical and histological abnormalities compared with hepatitis C patients without alcohol abuse. ${ }^{14}{ }^{15}$ 17-20 Poynard et al showed that patients who consumed more than $50 \mathrm{~g}$ of alcohol daily had a 34\% increased rate of fibrosis progression compared with nondrinkers. ${ }^{17}$ Other workers have also noted increased hepatic fibrosis, cirrhosis, end stage liver disease, and death in HCV patients who consume alcohol. ${ }^{18-20}$ The mechanism by which alcohol worsens HCV related liver disease is not clear. Several workers have assessed the influence of alcohol on HCV viral replication, with variable results. ${ }^{13171821-31}$ Some workers have noted increased viral levels in alcoholics ${ }^{17} 22270$ while others have found no difference between drinkers and abstinent individuals. ${ }^{18} 23-26282931$ Similarly, the effect of abstinence in the same individual was associated with either no effect ${ }^{28}$ or a decrease in viral titres. ${ }^{23}{ }^{24}$ The inconsistent results can be explained by factors such as varying definitions of grading alcohol consumption, duration of alcohol use, presence or absence of active drinking at the time of enrolment, and duration of abstinence prior to the study. In view of these difficulties, we carried out a systematic review and meta-analysis of all existing studies to determine whether or not alcohol consumption has a stimulatory effect on hepatitis $\mathrm{C}$ virus levels.

\section{MATERIALS AND METHODS \\ Data collection}

A literature search for all studies from 1989, when HCV was first identified, to the present (2004) was performed using the PubMed, MEDLINE, and Cochrane databases. A variety of medical subject headings were used including alcohol, alcohol abuse, alcoholism AND (hepatitis C virus titres OR viral levels OR RNA, OR quantitative assay). The search was limited to human studies but included full publications, abstracts, and non-English language articles. In addition, a manual search was conducted of all related publications and review articles. The relevant studies were assessed for the following information: alcohol consumption (g/day), information on whether subjects were actively drinking at the

Abbreviations: $\mathrm{HCV}$, hepatitis $\mathrm{C}$ virus; bDNA, branched DNA; PCR, polymerase chain reaction 
time of analysis, methods used to measure HCV titres, and mean values and standard deviations of viral titres. Quantitative HCV assay was performed by two different techniques in the studies: eight studies used the branched DNA (bDNA) methodology while the remaining six studies employed the quantitative polymerase chain reaction (PCR) technique. The bDNA assay expresses the results as genome milliequivalents per $\mathrm{ml}(\mathrm{mEq} / \mathrm{ml})$ and has two different versions of the test. The initial version (Quantiplex 1.0) had a lower limit of detection of 350000 viral $\mathrm{mEq} / \mathrm{ml}$ while the next version of the assay (Quantiplex 2.0) had a lower limit of detection of $200000 \mathrm{viral} \mathrm{mEq} / \mathrm{ml}$. The results of the PCR assay are expressed as $\log _{10}$ copies of $\mathrm{RNA} / \mathrm{ml}$ of serum.

\section{Data analysis}

The effect of alcohol on viral titres was investigated in three different ways. The primary analysis focused on comparing the heaviest drinking group with the non-drinking group in each study, whenever the data provided allowed such a comparison. The second analysis examined the effect of graded doses of alcohol based on studies that divided subjects into non-drinkers, moderate drinkers, and heavy drinkers. Finally, we assessed the effect of abstinence in the same individual. Not all of the requisite data were available in some studies but it was possible to make estimates based on other information, as described in the results section.

To assess the effect of heavy alcohol abuse, confidence intervals and $\mathrm{p}$ values for both individual and combined studies were based on our calculations, assuming normal distributions with equal population standard deviations for each group within studies, although such assumptions were not made by the authors in at least some of the studies. It is reasonable to assume an approximate normal distribution for both groups in most studies because of adequate sample sizes, especially when the standard deviations are not excessively large in comparison with the mean values. Whenever direct comparisons could be made to the authors' confidence intervals and p values, we found quite close agreement. Also, the meta-analysis calculations are based only on the effect size and its variance in each study, and the variance is a function of only the effect size itself and the sample size in each group. Comparisons between groups, as reported by the studies, were based on Wilcoxon rank sum tests, Student's $t$ tests, or analyses of variance followed by $t$ tests. As our calculations of $p$ values were based on normality assumptions, they were not identical to those reported in studies in which significance was based on the Wilcoxon test. However, there was complete agreement with respect to statistical significance (that is, whether or not $\mathrm{p}<0.05$ ).

In order to combine the results from the individual studies it was necessary to convert the data to a common metric. This was accomplished by defining an "effect size" for each study by dividing the difference between mean values by the pooled estimate of standard deviation. As the mean values and standard deviations are expressed in the same units of measurement, their ratio is independent of the measurement units. Thus, for example, the studies based on bDNA can be directly assessed along with those based on PCR assay as long as both methods are designed to measure the same phenomenon. A positive effect size indicates that the heavy drinking group had a higher viral load than the non-drinking group.

The variance (Var) of each effect size was calculated by the formula:

Var $=\left(\mathrm{n}_{1}+\mathrm{n}_{2}\right) /\left(\mathrm{n}_{1} \times \mathrm{n}_{2}\right)+\mathrm{ES}^{2} /\left(2 \times\left(\mathrm{n}_{1}+\mathrm{n}_{2}\right)\right)$

where $n_{1}$ and $n_{2}$ are the sample sizes of the two groups being examined (for example, non-drinking and heavy drinking groups, respectively) and "ES" represents the effect size. A computer program designed to combine data from separate studies, including input statements, was used, as described by Cooper and Hedges. ${ }^{32}$ Data analysis was performed on the SAS statistical software program (version 8; SAS Institute Inc., Cary, North Carolina, USA). Input to the analysis included the individual effect sizes and their variances. Output included the combined estimate of effect size, its standard deviation and confidence limits, and $p$ value based on a "fixed effects" model, along with a test of heterogeneity among the various studies. Heterogeneity would indicate that the studies may not all be estimating the same quantity, and would question the legitimacy of the fixed effects model assumptions. Also included in the output were combined results based on a "random effects" model that is appropriate when the results of individual studies are considered to represent estimates of differing effects.

\section{RESULTS}

\section{Study material}

A total of 14 studies were identified ${ }^{13171821-31}$ (table 1). Five of these studies ${ }^{1321} 232731$ did not provide actual viral titres. Of these five, two studies did not provide any data at all. The remaining three studies ${ }^{23} 273$ noted the difference in viral levels between drinkers and abstinent subjects without

\begin{tabular}{|c|c|c|c|c|}
\hline \multirow[b]{2}{*}{ Author } & \multirow[b]{2}{*}{ Method } & \multicolumn{2}{|l|}{ Viral titres } & \multirow[b]{2}{*}{ p Value } \\
\hline & & Non-drinkers (n) & Heavy drinkers (n) & \\
\hline Takase $^{13}$ & PCR & No data & No data & - \\
\hline Sawada ${ }^{21}$ & bDNA & No data & No data & - \\
\hline Oshita $^{22}$ & PCR & $7.7(0.8)(37)$ & $8.5(0.5)(16)$ & $<0.01$ \\
\hline Cromie $^{23}$ & PCR & No data & No data & NS \\
\hline $\mathrm{Sata}^{24}$ & bDNA & $15.4(13.9)(15)$ & $16.5(49.4)(20)$ & NS \\
\hline Ohnishi ${ }^{25}$ & PCR & $8.0(1.6)(35)$ & $7.2(2.0)(10)$ & NS \\
\hline Poynard"7 & bDNA-2 & $4.7(7.2)(105)$ & $8.9(6.8)(16)$ & 0.03 \\
\hline Yoshihara ${ }^{26}$ & PCR & 4.9 (1.6) (33) & $4.4(1.6)(14)$ & NS \\
\hline Pessione $^{27}$ & bDNA-2 & No data & No data & 0.001 \\
\hline Wiley $^{18}$ & bDNA & $5.9(10.0)(44)$ & $5.7(6.4)(28)$ & NS \\
\hline Anand $^{28}$ & bDNA-1 and 2 & $54.4(66.8)(18)$ & $78.4(112.8)(50)$ & NS \\
\hline $\operatorname{Khan}^{29}$ & bDNA-1 & $2.7(2.9)(40)$ & $2.3(1.7)(24)$ & NS \\
\hline Loguercio $^{30}$ & bDNA-2 & $0.6(0.3)(17)$ & $14.9(6.3)(17)$ & $<0.01$ \\
\hline Romero-Gomez ${ }^{31}$ & PCR & No data & No data & NS \\
\hline
\end{tabular}

bDNA, branched DNA technique (numbers after bDNA refer to the first or second generation of the test); PCR, polymerase chain reaction assay. For the purpose of uniformity, all results were converted to mean $(S D)(\times 10)$ to the sixth power. All $p$ values are as reported by the studies reviewed. 
Table 2 Effect of highest alcohol consumption on hepatitis $C$ viral levels compared to non-drinkers. The results are arranged in order of decreasing effect

\begin{tabular}{|c|c|c|c|c|c|c|}
\hline Author & N1 & N2 & Effect size & Variance & $95 \% \mathrm{Cl}$ & p Value \\
\hline Loguercio $^{30}$ & 17 & 17 & +3.21 & 0.27 & +2.17 to +4.25 & $<0.001$ \\
\hline Oshita 22 & 37 & 16 & +1.10 & 0.10 & +0.47 to +1.74 & $<0.001$ \\
\hline Poynard ${ }^{17}$ & 105 & 16 & +0.59 & 0.07 & +0.05 to +1.13 & 0.03 \\
\hline Anand $^{28}$ & 18 & 50 & +0.24 & 0.08 & -0.31 to +0.79 & 0.39 \\
\hline $\mathrm{Sata}^{24}$ & 15 & 20 & +0.03 & 0.12 & -0.67 to +0.72 & 0.93 \\
\hline Wiley $^{18}$ & 44 & 28 & -0.02 & 0.06 & -0.50 to +0.46 & 0.92 \\
\hline Khan $^{29}$ & 40 & 24 & -0.16 & 0.07 & -0.67 to +0.36 & 0.54 \\
\hline Yoshihara $^{26}$ & 33 & 14 & -0.31 & 0.10 & -0.96 to +0.33 & 0.33 \\
\hline Ohnishi ${ }^{25}$ & 35 & 10 & -0.48 & 0.13 & -1.20 to +0.24 & 0.19 \\
\hline \multicolumn{7}{|l|}{ Combined studies } \\
\hline Fixed effects model & & & +0.24 & 0.010 & +0.05 to +0.45 & 0.01 \\
\hline Random effects model & & & +0.43 & 0.143 & -0.31 to +1.17 & 0.29 \\
\hline Test for heterogeneity & & & & & & $<0.001$ \\
\hline
\end{tabular}

providing actual viral titres; two of these studies found no difference between subjects who consumed alcohol and nondrinkers ${ }^{23}{ }^{31}$ while the third ${ }^{27}$ noted significantly higher viral titres in alcohol users $(\mathrm{p}<0.001)$. The remaining nine studies provided more complete data.

\section{Effect of heavy alcohol abuse}

The pertinent data from the individual studies, along with combined results, are shown for both the fixed effects and random effects models in table 2 . Three studies found a significant positive association between alcohol consumption and virus load. ${ }^{17} 2230$ Another two studies showed a positive effect $^{24} 28$ while the remaining four studies ${ }^{18} 252629$ found a negative relationship (table 2). In the analysis of the combined results using the fixed effects and random effects models, the test for heterogeneity among studies was highly significant, indicating that not all studies were estimating the same effects. Consequently, the fixed effects model was not appropriate as it is based on the assumption that there are no real differences in any methodologies across studies. Therefore, our conclusions are based appropriately on the random effects model which relaxes the requirement of identical characteristics among studies. Based on the random effects model there was no conclusive evidence that alcohol consumption is associated with increased HCV viral titres $(\mathrm{p}=0.29)$.

In order to further analyse the results, we performed a sensitivity test by deleting the study by Loguercio and colleagues $^{30}$ from the combined analysis. This was designed to determine the degree to which the results would change if the study with greatest positive effect was not included. As a result, the fixed effects model was now no longer statistically significant. As before, the random effects model was not statistically significant and the test for heterogeneity among studies was highly significant $(p=0.008)$. These findings reinforced the interpretation that the nine studies selected for the meta-analysis do not all estimate the same population effect size. Rather, there were unknown differences among the studies accounting for the heterogeneity of results among them.

\section{Effect of graded doses of alcohol}

Only four studies divided drinking subjects into moderate drinking and heavy drinking groups. The results are shown on table 3. The cut off for moderate and heavy drinking varied in the four studies. In one study, ${ }^{30}$ alcohol intake was divided into three categories: $<40 \mathrm{~g} / \mathrm{day}, 41-80 \mathrm{~g} / \mathrm{day}$, and $>80 \mathrm{~g} /$ day. HCV RNA levels were comparable for the two lower categories which were combined. In another study, ${ }^{17}$ moderate and heavy drinking were defined as $<50 \mathrm{~g} /$ day and $>50 \mathrm{~g} /$ day, respectively. In the third study, ${ }^{26}$ the categories were $<46 \mathrm{~g} /$ day and $>46 \mathrm{~g} /$ day, respectively. The fourth study divided the drinking groups into $<80 \mathrm{~g} /$ day and $>80 \mathrm{~g} /$ day. Within each study and for the combined analysis we performed pairwise comparisons of effects between the nondrinking subjects and the two drinking groups. In two studies, ${ }^{17}{ }^{30}$ there was an increasing effect on viral titres with higher doses of alcohol. The third study ${ }^{26}$ showed the same effect for non-drinkers and moderate drinkers with a decreasing effect for heavy drinkers. The fourth study ${ }^{29}$ gave the same decreased effect for moderate and heavy drinkers. Basing our choice of model on the test for heterogeneity, there was no significant difference between non-drinkers and moderate drinkers $(p=0.50$; fixed effects model), between non-drinkers and heavy drinkers $(p=0.35$; random effects model), or between moderate drinkers and heavy drinkers $(p=0.32$; random effects model). For this analysis the requisite data for study by Loguercio and colleagues $^{30}$ was

Table 3 Effect of graded doses of alcohol consumption on hepatitis $C$ virus titres. The study groups consist of non-drinkers (1), moderate drinkers (2), and heavy drinkers (3)

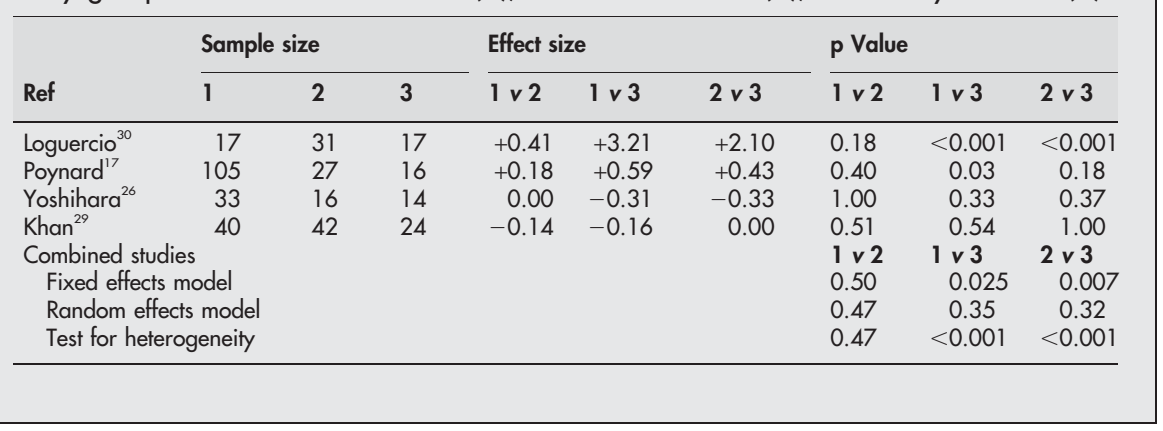


Table 4 Hepatitis C virus (HCV) titres in the same individuals at baseline and after a period of abstinence

\begin{tabular}{lllllll}
\hline & \multirow{2}{*}{$\begin{array}{l}\text { Subject } \\
\text { Author }\end{array}$} & No & Method & $\begin{array}{l}\text { Duration of } \\
\text { abstinence }\end{array}$ & Viral titres & \\
& & & Baseline & Abstinence & p Value \\
\hline Takase $^{13}$ & 9 & PCR & Unclear & NA & NA & HCV-ve in 4 of 8 \\
Sawada $^{21}$ & 11 & bDNA & 4 weeks & NA & NA & HCV fell in 5 of 11 \\
Cromie $^{23}$ & 12 & PCR & $4.4(0.2)$ mo & NA & NA & 0.035 \\
Sata $^{24}$ & 24 & bDNA & $30-90$ days & $16.5 \times 10$ & $2.84 \times 10$ & 0.01 \\
Anand $^{28}$ & 3 & bDNA & 6 mo & $108(111.3)$ & $98(78.7)$ & NS \\
\hline
\end{tabular}

Statistical analysis of the combined studies was not possible because none of the studies provided the mean and SD of change in viral load for individual subjects at baseline and after abstinence.

bDNA, branched DNA technique. bDNA results expressed as viral $\mathrm{mEq} / \mathrm{ml}$.

$\mathrm{PCR}$, polymerase chain reaction assay; NA, not available; mo, months.

estimated from a graph as the actual values were not provided.

\section{Effect of abstinence}

Five studies assessed the same individuals before and after a period of abstinence. ${ }^{1321232428}$ The results are shown in table 4. In all of the studies the number of subjects included were small. In three studies viral levels were not provided: in one of these studies, ${ }^{13}$ HCV markers disappeared in four of eight patients $(50 \%)$ after abstinence; in the second ${ }^{21}$ viral titres fell in five of $11(45 \%)$ and remained the same or increased in the remaining six patients; while in the third study abstinance was associated with a significant fall in viral load. ${ }^{23}$ There were discordant findings in the two studies where actual titres were available: one study ${ }^{24}$ showed a significant fall in viral load after abstinence while the other found no difference. ${ }^{28}$ Statistical analysis of the combined studies was not possible because none of the studies provided the necessary mean and SD of change in viral load before and after abstinence.

\section{Effect of HCV quantitative assays}

In order to assess whether the differences in HCV quantitative methodologies did not account for the lack of significance, we performed separate analyses for the six bDNA and three PCR studies and found that both failed the heterogeneity test, their effect sizes under the random model were non-significant $(\mathrm{p}=0.30$ and $\mathrm{p}=0.82$, respectively), and the difference between the two random models was also not significant $(p=0.50)$.

\section{DISCUSSION}

The present study was carried out to determine the influence of alcohol consumption on HCV viral levels. Several problems were encountered in conducting the meta-analysis. Firstly, there was little uniformity in different studies in grading the severity of alcohol consumption. The division between modest and high alcohol use, based on daily consumption, showed wide variation: $10 \mathrm{~g}^{22} 40 \mathrm{~g},{ }^{18} 46 \mathrm{~g}^{26} 50 \mathrm{~g}^{,{ }^{17}}$ and 80 g. $^{28}{ }^{29}$ In one study, subjects were divided into two groups of high alcohol consumption: $23-69 \mathrm{~g} /$ day and $>69 \mathrm{~g} /$ day. ${ }^{25}$ In some studies, patients were divided into drinkers and abstinent groups, but the amount of daily consumption was not noted. However, the primary consideration in assessing the effect of alcohol was to compare a group of heavy alcohol consumers with complete abstainers or minor users of alcohol. Fortunately, in all of the studies included in the meta-analysis such a division was possible. There is no question in all the studies that the heaviest user group qualified to be thereby defined, and if there was a relationship between alcohol consumption and viral titre it should emerge. Another area of difficulty was the use of two different methodologies for measurement of serum viral titres, which express results differently: $\mathrm{mEq} / \mathrm{ml}$ with the bDNA technique and $\log$ copies of RNA/ml with the PCR assay. Although the values provided by the two assays cannot be exactly equated, they represent a similar spectrum in serum viral titres. The advantage of meta-analysis is that it takes into account the disparities in different studies. Moreover, by defining the "effect size" for each study (obtained by dividing the difference between mean values by the pooled estimate of standard deviation), data obtained by the bDNA assay could be directly compared with the PCR assay as the effect size is then independent of the measurement units and both methods were designed to measure the same phenomenon. Finally, it should also be emphasised that studies based on patient recall of alcohol consumption have an inherent fundamental problem and a meta-analysis cannot completely exclude the possibility that a true positive relationship between alcohol use and viral titres does or does not exist. The only way to conclusively assess the effect of graded doses of alcohol on HCV viral replication is to perform a controlled prospective study using validated questionnaires of life time alcohol consumption. However, we believe that in the absence of such data, the next best approach is collective analysis of different studies by meta-analysis.

Our findings indicate that alcohol consumption does not influence serum hepatitis $\mathrm{C}$ virus levels. We analysed the influence of alcohol use in three different ways. Firstly, we compared hepatitis $\mathrm{C}$ viral titres between the group categorised as the heaviest drinkers in each study and abstinent subjects (table 2). The combined analysis based on the random effects model showed no conclusive evidence $(\mathrm{p}=0.29)$ to support the fact that alcohol consumption is associated with increased viral titres. Assessment of graded doses of alcohol was performed by dividing subjects into three groups: complete abstainers, moderate alcohol users, and heavy alcohol users, based on the authors' assessments. Meta-analysis could be performed in only four studies where such data were available (table 3). Again, based on the test for heterogeneity, there was no significant difference between non-drinkers and moderate drinkers $(\mathrm{p}=0.50$; fixed effects model), between non-drinkers and heavy drinkers $(\mathrm{p}=0.35$; random effects model), or between moderate drinkers and heavy drinkers $(p=0.32$; random effects model). We also examined the effect of abstinence on HCV levels in the same individual. The number of subjects enrolled in all of the studies was small. There were discordant findings among the studies and statistical analysis of the combined data could not be performed because none of the studies provided the mean and standard deviation for change of viral titres before and after abstinence.

The question arises as to why uniform results were not obtained in different studies. One possibility is the methodology (bDNA or PCR) used to quantitate HCV viral load. However, this factor is unlikely to be important because we 
performed separate analyses for the bDNA and PCR studies and found that both failed the heterogeneity test, their effect sizes under the random model were not significant, and the difference between the two random models was also not significant. Moreover, one of the PCR based studies found the second to largest positive effect size while the other two found the largest negative effect sizes. Another potential cause for lack of uniformity could be the timing of the HCV assay with respect to alcohol consumption. Fortunately, most studies included in the meta-analysis comprised subjects who were "current" alcohol users, implying recent or active consumption as opposed to past alcohol use. We also considered the possibility of publication bias, which again failed to indicate any explanation for the differences among studies. We believe the only plausible explanation is the relatively small sample size employed in the different studies, a defect that is overcome to some extent by the use of metaanalysis.

In conclusion, the present study fails to support the theory that alcohol consumption has a stimulatory effect on HCV levels in serum. It is possible that alcohol may influence intrahepatic viral kinetics. However, in the absence of such data, it is tempting to postulate that the damaging effect of ethanol and HCV is simply additive and may involve different mechanisms and signalling pathways.

\section{Authors' affiliations}

B S Anand, J Thornby, Department of Medicine, Michael E DeBakey VA Medical Center and Baylor College of Medicine, Houston, Texas, USA

Conflict of interest: None declared.

\section{REFERENCES}

1 Alter MJ, Kniszon-Moran D, Nainan OV, et al. The prevalence of hepatitis C in the United States, 1988 through 1994. N Engl J Med 1999;341:556-62.

2 Hoofnagle JH. Course and outcome of hepatitis C. Hepatology 2002;36:S21-9.

3 Thomas DL. Hepatitis $C$ and human immunedeficiency virus infection. Hepatology 2002;36(suppl 1):S201-9.

4 Vogt M, Lay T, Frosner G, et al. Prevalence and clinical outcome of hepatitis C infection in children who underwent cardiac surgery before implementation of blood-donor screening. N Engl J Med 1999:341:866-70.

5 Poynard T, Ratzui V, Charlotte F, et al. Rates and risk factors of liver fibrosis progression in patients with chronic hepatitis C. J Hepatol 2001;34:764-7.

6 Deuffic S, Buffat L, Poynard T, et al. Modeling the hepatitis C virus epidemic in France. Hepatology 1999;29:1596-601.

7 Bissel DM. Sex and hepatic fibrosis. Hepatology 1999;29:988-9.

8 Wiley TE, Brown J, Chan J. Hepatitis C virus infection in African Americans: its natural history and histological progression. Am J Gastroenterol 2002;97:700-6

9 Harris DR, Gonin R, Alter HJ, et al. The relationship of acute transfusionassociated hepatitis to the development of cirrhosis in the presence of alcohol abuse. Ann Intern Med 2001;134:120-4.
10 Jeffers L, Cassidy W, Howell CD, et al. Peginterferon alfa-2a (40 kd) and ribavirin for Black American patients with chronic HCV genotype 1. Hepatology 2004;39:1702-8.

11 Chuang WL, Chang WY, Lu SN, et al. The role of hepatitis $C$ virus in chronic hepatitis B virus infection. Gastroenterol Jpn 1993;28(suppl 5):23-7.

12 Tsai JF, Jeng JE, Ho MS, et al. Independent and additive effect modification of hepatitis $B$ and $C$ virus infection on the development of chronic hepatitis. J Hepatol 1996;24:271-6.

13 Takase S, Takada N, Sawada M, et al. Relationship between alcoholic liver disease and HCV infection. Alcohol Alcohol 1993;28:77-84.

14 Coelho-Little ME, Jeffers $\sqcup$, Bernstein DE, et al. Hepatitis $C$ virus in alcoholic patients with and without clinically apparent liver disease. Alcohol Clin Exp Res 1995; 19:1173-6.

15 Rosman AS, Waraich A, Galvin K, et al. Alcoholism is associated with hepatitis $C$ but not hepatitis B in an urban population. Am J Gastroenterology 1996:91:498-505.

16 Nevins $\mathrm{CL}$, Malaty $\mathrm{H}$, Velez ME, et al. Interaction of alcohol and hepatitis $\mathrm{C}$ virus infection on severity of liver disease. Dig Dis Sci 1999;44:1236-42.

17 Poynard T, Bedrossa P, Opolon P. Natural history of liver fibrosis progression in patients with chronic hepatitis C. The OBSVIRIC, METAVIR, CLINIVIR, and DOSVIRIC groups. Lancet 1997;349:825-32.

18 Wiley TE, McCarthy M, Breidi L, et al. Impact of alcohol on the histological and clinical progression of hepatitis $C$ infection. Hepatology 1998;28:805-9.

19 Thomas DL, Astemborski J, Rai RM, et al. The natural history of hepatitis C virus infection: host, viral, and environmental factors. JAMA 2000;284:450-6

20 Monto A, Patel K, Bostrom A, et al. Risks of a range of alcohol intake on hepatitis C-related fibrosis. Hepatology 2004;39:826-34

21 Sawada M, Takada A, Takase S, et al. Effects of alcohol on the replication of hepatitis C virus. Alcohol Alcoholism 1993;28:85-90.

22 Oshita M, Hayashi N, Kasahara A, et al. Increased serum hepatitis C virus RNA levels among alcoholic patients with hepatitis $C$. Hepatology 1994;20:1115-20.

23 Cromie SL, Jenkins PJ, Bowden S, et al. Chronic hepatitis C: effect of alcohol on hepatic activity and viral titer. J Hepatol 1996;25:821-6.

24 Sata $M$, Fukuizumi $K$, Uchimura $Y$, et al. Hepatitis $C$ virus infection in patients with clinically diagnosed alcoholic liver disease. J Viral Hepat 1996;3:143-8.

25 Ohnishi K, Matsuo S, Matsutani K, et al. Interferon therapy for chronic hepatitis $C$ in habitual drinkers: Comparison with chronic hepatitis $C$ in infrequent drinkers. Am J Gastroenterol 1996;91:1374-9.

26 Yoshihara H, Noda K, Kamada T. Interrelationship between alcohol intake, hepatitis C, liver cirrhosis, and hepatocellular carcinoma. Recent Dev Alcohol 1998; 14:457-69.

27 Pessione F, Degos F, Marcellin P, et al. Effect of alcohol consumption on serum hepatitis $C$ virus RNA and histological lesions in chronic hepatitis C. Hepatology 1998;27:1717-22

28 Anand BS, Velez M. Influence of chronic alcohol abuse on hepatitis $C$ virus replication. Dig Dis 2000;18:168-71.

29 Khan KN, Yatsuhashi H. Effect of alcohol consumption on the progression of hepatitis $C$ virus infection and risk of hepatocellular carcinoma in Japanese patients. Alcohol Alcohol 2000;35:286-95.

30 Loguercio C, Di Pierro M, Di Marino MP, et al. Drinking habits of subjects with hepatitis $C$ virus-related chronic liver disease: Prevalence and effect on clinical, virological and pathological aspects. Alcohol Alcohol 2000;35:296-301.

31 Romero-Gomez M, Grande L, Nogales MC, et al. Intrahepatic hepatitis C virus replication is increased in patients with regular alcohol consumption. Dig Liver Dis 2001;33:698-702.

32 Cooper H, Hedges L, eds. The handbook of research synthesis. New York: The Russell Sage Foundation, 1994. 